\title{
Work of the planetary gear trains as differentials and their capabilities
}

\author{
Kiril Arnaudov ${ }^{1}$, Stefan Petrov $^{2}$, and Emiliyan Hristov ${ }^{2}$ \\ ${ }^{1}$ Institute of Mechanics, Bulgarian Academy of Sciences, Sofia, Bulgaria \\ ${ }^{2}$ Podemcrane AD, Gabrovo, Bulgaria
}

\begin{abstract}
Planetary gear trains can work differently, namely, with $\mathrm{F}=1$ degree of freedom, i.e. as reducers or multipliers, and also with $\mathrm{F}=2$ degrees of freedom, i.e. as differentials. Moreover, with a two-motor drive they work as a summation planetary gear train and with a one-motor drive, they work as a division planetary gear train. The most popular application of planetary gear trains is as a differential which is bevel and is produced globally in millions of pieces. Some of the cylindrical planetary gear trains can also be used as differentials. Although less often, they are used in heavy wheeled and chain vehicles such as trailer trucks, tractors and tanks. They are also very suitable for lifting machines with a two-motor drive which provides maximum security for the most responsible cranes, such as the metallurgical ones. Initially the paper presents some simple, i.e. single-carrier cylindrical planetary gear trains, both with external and internal meshing, driven by 2 motors. Their kinematic capabilities and velocity, respectively, are considered to realize the necessary gear ratio. Finally, the case of a compound two-carrier planetary gear train is considered, which is composed of 2 simple planetary gear trains. This shows that not only the simple planetary gear trains, i.e. the single-carrier ones, can work as differentials.
\end{abstract}

\section{Introduction}

When it comes to differentials, no doubt what usually comes to mind is the automobile differential (Fig. 1, a), which is by definition bevel and produced annually worldwide in millions of pieces by the automotive industry. The car differential is characterized by a single motor drive and thus it operates as a division gear train. The movement that the carrier receives from the motor is split towards the two executive elements - the two driving wheels.

There is another type of differential, the cylindrical differential, which, although less often, is used in vehicles (Fig. 1, b), and not only there. Both types of differentials are referred to as symmetrical, since (ignoring the friction losses) they transmit the same torques from the carrier to the two shafts connected to the two central gear wheels, which drive the two wheels or tracks of the respective vehicle - trailer trucks, tractors and tanks. In addition to vehicles, these differentials are used to provide maximum security in lifting equipment, in metallurgical cranes, etc. In such cases, these differentials are driven by a two-motor drive, i.e. they work as a summation gear train. The two motors most often have the same power and angular velocity, so that in case of failure of one of the motors, the other one continues to work with reduced velocity.

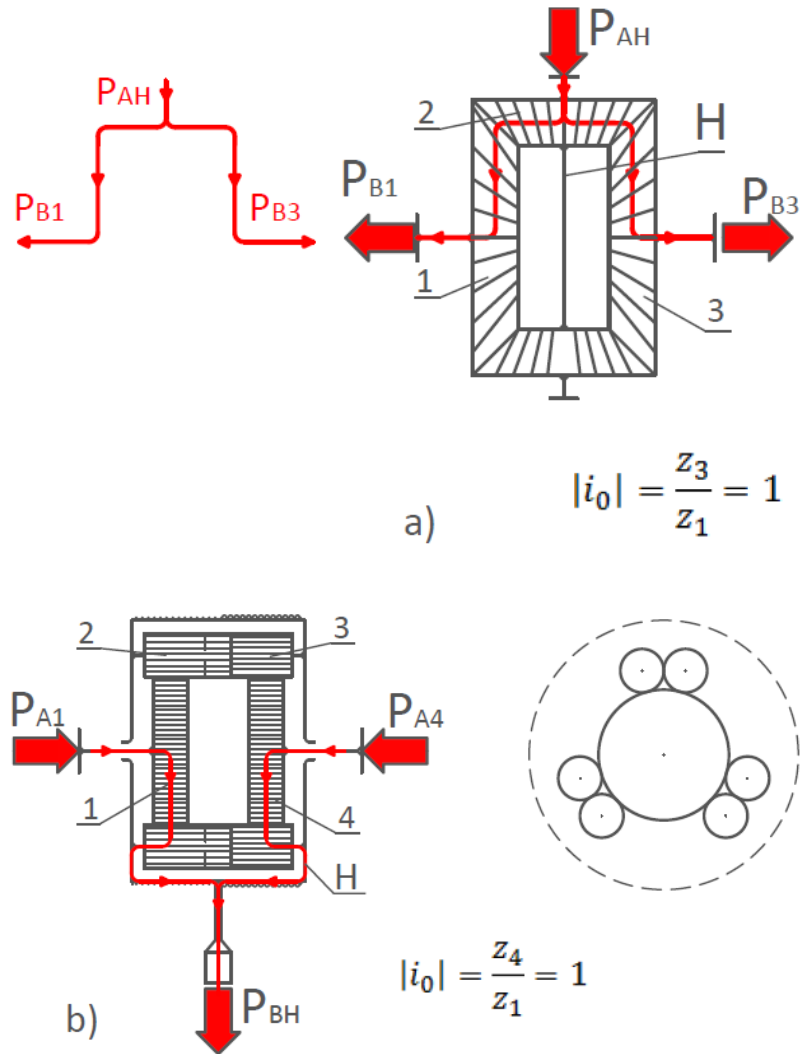

Fig. 1. Bevel (a) and cylindrical (b) differential: $\mathrm{P}_{\mathrm{A}}-$ input power; $\mathrm{P}_{\mathrm{B}}-$ output power.

Corresponding author: $\mathrm{k}$ arnaudov@abv.bg 


\section{Objective}

The objective of this paper is to review and analyze the planetary gear train types which are suitable for working with $\mathrm{F}=2$ degrees of freedom, i.e. as differentials, and to reveal the capabilities they have. Under consideration are the planetary gear trains with cylindrical rather than the bevel gear wheels. These are initially 4 types of simple, i.e. single-carrier planetary gear trains:

$\overline{\mathrm{AAA}}$ - planetary gear traisan with 3 meshings, all of which are external (Fig. 2);

$\overline{\text { IAI }}$ - planetary gear train with 3 meshings, two of which are internal, and the third one is external (Fig. 4);

$\overline{\mathrm{AI}}$ - planetary gear train with 2 meshings, one of which is external, and the other one is internal (Fig. 5);

$\overline{\mathrm{AAI}}$ - planetary gear train with 3 meshings, two of which are external, and the third one is internal (Fig. 6).

The indices of these types of simple planetary gear trains are according to [1]. The letters $\mathrm{A}$ and I refer to the external and the internal meshings, respectively. Later on these differentials are considered only as summation gear trains, i.e. with a two-motor drive.

In addition to the simple planetary gear trains, at the end of the paper the case of a compound planetary gear train is considered, which can also work as a differential (Fig. 7).

\section{$3 \overline{\mathrm{AAA}}$ - planetary gear train}

Fig. 2 shows three versions of this gear train. When the two central gear wheels 1 and 4 have equal number of teeth, i.e.

$$
z_{1}=z_{4}
$$

the basic speed ratio $i_{o}$ of the gear train is

$$
i_{0}=\frac{\omega_{1}}{\omega_{4}}=-\frac{z_{4}}{z_{1}}=-1
$$

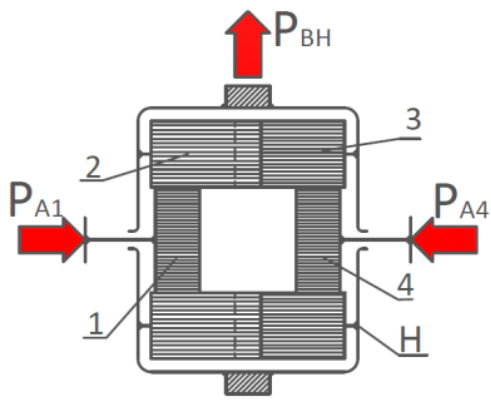

a)

$i_{0}=\frac{\omega_{1}}{\omega_{4}}=-\frac{z_{4}}{z_{1}}=-1 ;\left(\mathrm{z}_{1}=\mathrm{z}_{4} !\right)$
In addition to the fact that most often with this gear train $z_{1}=z_{4}$ (Fig. 2, a), the two driving central gear wheels 1 and 4 have the same power $P_{1}=P_{4}$ and the same angular velocity $\omega_{1}=\omega_{4}$.

When the two motors rotate unidirectionally, the gear train rotates as a unit, and the angular velocity $\omega_{H}$ of the carrier $\mathrm{H}$, which is the output element, is the same as that of the motors, i.e.

$$
\omega_{B}=\omega_{H}=\omega_{A}
$$

Here and below the indices A and B denote the input and the output elements, respectively.

If for any reason one of the motors fails, then at basic speed ration $i_{0}=-1$, the other motor continues to work and the carrier $\mathrm{H}$ continues to rotate but at an angular velocity reduced in half, i.e.

$$
\omega_{B}=\omega_{H}=0.5 \omega_{A} .
$$

In the first considered version of the gear train, where $\mathrm{i}_{0}=-1$, no reverse rotation of the two motors is performed, since in this case the carrier $\mathrm{H}$ remains still, i.e.

$$
\omega_{B}=\omega_{H}=0 .
$$

The capabilities described here are not the only ones that the $\overline{\mathrm{AAA}}$ - gear train can present. The other two versions have bigger capabilities (Fig. 2, b and c). When the number of teeth of the two central gear wheels 1 and 4 is not the same, i.e.

$$
z_{1}<z_{4}
$$

and the basic speed ration of the gear train is not $i_{0}=-1$, but

$$
i_{0}=\frac{\omega_{1}}{\omega_{4}}=-\frac{z_{4}}{z_{1}}<-1, \text { respectively }\left|i_{0}\right|>1,
$$

the two versions of the gear train can perform not 2 , but 4 velocities in one direction.

Fig. 2. $\overline{A A A}$ - planetary gear train working as a differential. 
Here, as in the first version of the gear train, when the two motors rotate unidirectionally, the gear train rotates as a unit, where the angular velocity $\omega_{\mathrm{H}}$ of the carrier $\mathrm{H}$ is the same as that of the motors, i.e.

$$
\omega_{B}=\omega_{H}=\omega_{A} .
$$

When only the small gear wheel 1 rotates, at an angular velocity $\omega_{1}=\omega_{1 \mathrm{~A}}$, then the output angular velocity $\omega_{\mathrm{H}}=\omega_{\mathrm{B}}$ of the carrier $\mathrm{H}$ is determined by the following known formula:

$$
\omega_{B}=\omega_{H}=\frac{\omega_{1 A}}{1-i_{0}}=\frac{\omega_{1 A}}{1+\frac{z_{4}}{z_{1}}} .
$$

Conversely, when only the big central gear wheel 4 rotates at the same angular velocity $\omega_{4}=\omega_{4 \mathrm{~A}}$ then the output angular velocity $\omega_{H}=\omega_{B}$ of the carrier $\mathrm{H}$ is determined by the following known formula [1]

$$
\omega_{B}=\omega_{H}=\frac{\omega_{4 A}}{1-\frac{1}{i_{0}}}=\frac{\omega_{4 A}}{1+\frac{z_{1}}{z_{4}}} .
$$

The last possible case is when both central gear wheels 1 and 4 rotate, but in reverse directions. Their movements are not summed up and the output angular velocity $\omega_{\mathrm{H}}=\omega_{\mathrm{B}}$ of the carrier $\mathrm{H}$ is determined by the following formula

$$
\omega_{B}=\omega_{H}=-\frac{\omega_{1 A}}{1+\frac{z_{4}}{z_{1}}}+\frac{\omega_{4 A}}{1+\frac{z_{1}}{z_{4}}} .
$$

Although at the two versions considered (Fig. 2, b and $\mathrm{c}$ ), the angular velocities of the two motors and of the central gear wheels 1 and 4 should be the same, i.e.

$$
\omega_{1}=\omega_{4}
$$

their powers must be different

$$
P_{4}>P_{1}
$$

Obviously, in all the versions and cases discussed here, the angular velocity $\omega_{\mathrm{H}}$ of the carrier $\mathrm{H}$ as an output element depends on the basic speed ratio $i_{0}$. This is illustrated on Fig. 3. It is seen that by selecting the basic speed ratio $i_{0} 4$ angular velocities $\omega_{\mathrm{H}}$ of the carrier $\mathrm{H}$ can be performed in one direction. It is not necessary that all four angular velocities $\omega_{\mathrm{H}}$ of the carrier $\mathrm{H}$ should be used in any case. It is quite possible that one maximum velocity is required which is realized by unidirectional rotation of the two motors and one single minimum velocity, for example 5, 10 or 20 times lower than that of the motors. The latter is achieved, as already stated, with the opposite (reverse) rotation of the two motors, and at appropriately selected basic speed ratio $i_{0}$.

Another case is also possible when all four angular velocities $\omega_{\mathrm{H}}$ of the carrier $\mathrm{H}$ are required, but they must be evenly distributed. For example, when basic speed ratio $i_{0}=1.5$ is used the following angular velocities are achieved (Fig. 3):

$$
\omega_{\mathrm{B}}=0.6 \omega_{\mathrm{A}} ; \omega_{\mathrm{B}}=0.4 \omega_{\mathrm{A}} ; \omega_{\mathrm{B}}=0.2 \omega_{\mathrm{A}} ;
$$

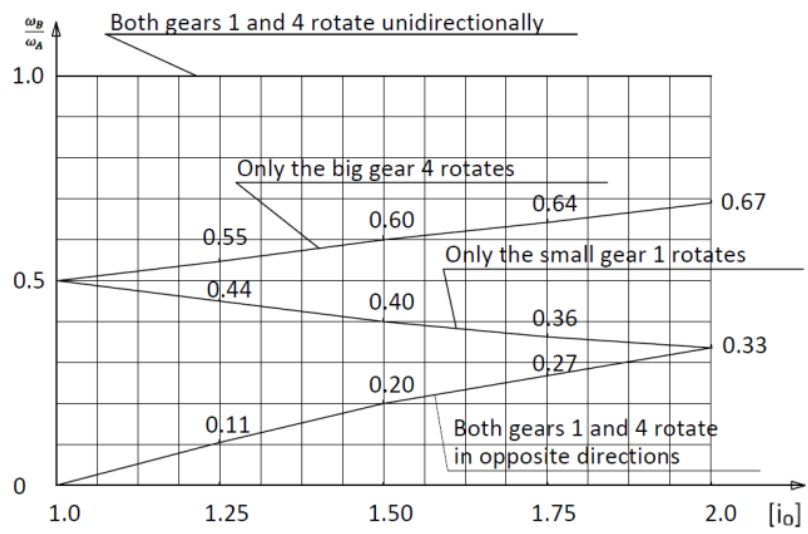

Fig. 3. Change of angular velocity $\omega_{H}=\omega_{B}$ of carrier $\mathrm{H}$ depending on the basic speed ratio $i_{0}$.

The $\overline{\mathrm{AAA}}$ - planetary gear train considered, working as a differential, has the advantage over the next type $\overline{\text { IAI }}$ - planetary gear train, in that it uses only gear wheels with external teeth. They are made easier and faster than the internal teeth. However, the bigger outer diameter which this gear train has, may in some cases turn to be a disadvantage.

\section{$4 \overline{\mathrm{IAI}}$ - planetary gear train}

This gear train (Fig. 4), although having different gear wheels as compared to the previous $\overline{\mathrm{AAA}}$ - gear train, namely, with internal teeth, and not with external ones, has the same kinematics as the $\overline{\mathrm{AAA}}$ - gear train, so that the diagram of Fig. 3 applies completely to it, too. Its basic speed ratio is the same when the number off teeth of the central gear wheels 1 and 4 is the same, i.e.

$$
z_{1}=z_{4}
$$

When this number of teeth is not the same, there are the same four cases as with the $\overline{\mathrm{AAA}}$ - gear train.

The $\overline{\text { IAI }}$ - gear train has the advantage that it can perform finer grading of the basic speed ratio $i_{0}$ of the gear train, and hence of the output angular velocity $\omega_{\mathrm{H}}$ of the carrier $\mathrm{H}$. This is due to the greater number of teeth $z_{1}$ and $z_{4}$ of the two central gear wheels 1 and 4, so that the gear train can perform even lower output angular velocity $\omega_{\mathrm{H}}$ as compared to $\overline{\mathrm{AAA}}-$ gear train. There is an interesting constructive opportunity to use only two planets (fig. 4, c). The small size of the gear train may also be considered an advantage. The internal teeth, which is technologically more complicated and more slow to produce, and also has some peculiarities, may be considered as a drawback [1-3]. 


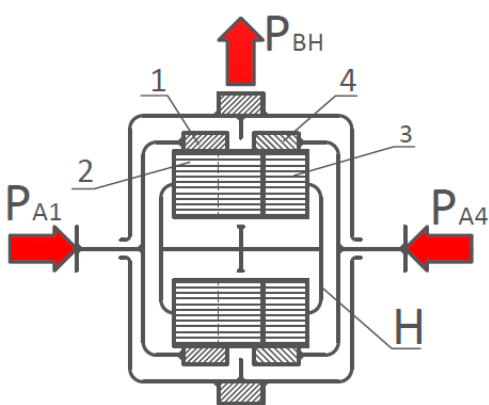

a)

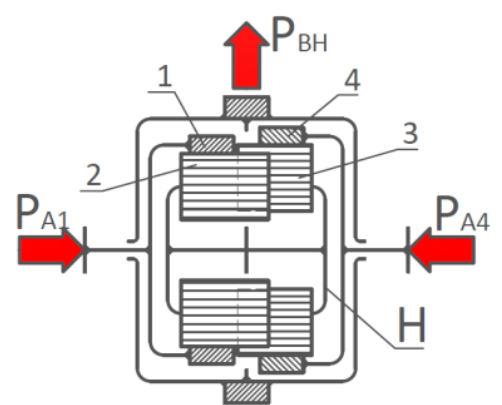

b)

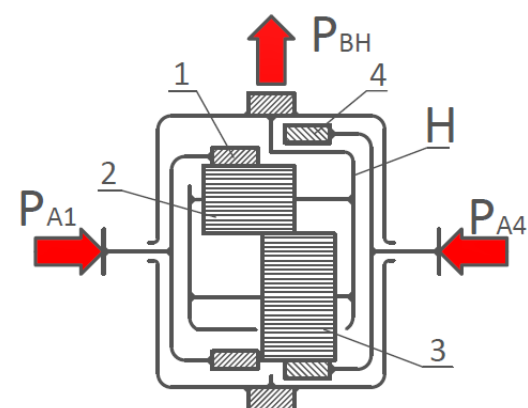

c)

$i_{0}=\frac{\omega_{1}}{\omega_{4}}=-\frac{Z_{4}}{Z_{1}}=-1 ;\left(\mathrm{z}_{1}=\mathrm{z}_{4} !\right)$

Fig. 4. $\overline{\text { IAI }}$ - planetary gear train working as a differential.

\section{$5 \overline{\mathrm{AI}}$ - planetary gear train}

This is the most often used type of planetary gear train (Fig. 5) and may also be used as a differential. With this gear train the two motors drive the two central gear wheels - the sun gear 1, which has external teeth and the ring gear 3 , which has internal teeth. The basic speed ratio of this gear train is

$$
i_{0}=\frac{\omega_{1}}{\omega_{3}}=-\frac{z_{3}}{z_{1}}<-1
$$

and it always has absolute value of

$$
\left|i_{0}\right|>1
$$

for example, bigger than $\left|i_{0}\right|>1.25$.

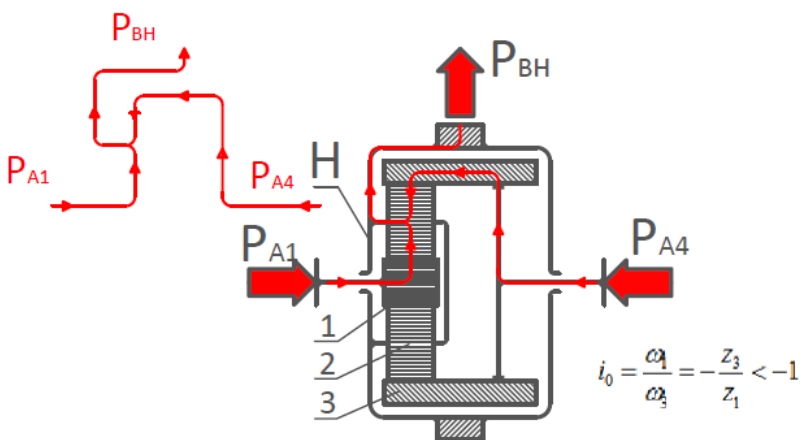

Fig. 5. $\overline{\mathrm{AI}}$ - planetary gear train working as a differential.

The diagram of Fig. 3 applies also to this gear train working as a differential, but it cannot perform angular velocity which is half of the angular velocity of the motors, when only one motor works. As it can be seen from the diagram, if the basic speed ratio $\left|i_{0}\right| \approx 1.25$, is considered to be the minimal one, this means that the gear train can perform approximately 10 times lower angular velocity $\omega_{H}$ of the carrier $\mathrm{H}$ than that of the motors, and $\omega_{1}$ and $\omega_{3}$ of the two central gear wheels, respectively. Although the two motors have the same angular velocity $\omega_{A}$, their power is not the same. It must be

$$
P_{3}>P_{1}
$$

The $\overline{\mathrm{AI}}$ - gear train considered, being the most often used planetary gear train, which has long been produced, no doubt offers the greatest security when used as a differential.

\section{$6 \overline{\mathrm{AAI}}$ - planetary gear train}

The design of this type of planetary gear train can be seen on Fig. 6. What is characteristic about it is that the two driving elements are different as compared to the previously described gear trains. These are the sun gear 1 and the carrier H. The output element is also different, and here it is ring gear 4 . The basic speed ratio is

$$
i_{0}=\frac{\omega_{1}}{\omega_{4}}=\frac{z_{4}}{z_{1}}>+1
$$

When $i_{0}=+2$ and the two motors rotate unidirectionally and drive the sun gear 1 and the carrier $\mathrm{H}$ with the same angular velocity $\omega_{\mathrm{A}}$, i.e.

$$
\omega_{1}=\omega_{H}=\omega_{A},
$$

then this gear train as the previous ones rotates as a unit, where the angular velocity $\omega_{4}$ of the ring gear 4 as an output element is the same as that of the motors, i.e.

$$
\omega_{B}=\omega_{4}=\omega_{A} .
$$

When only the sun gear 1 or only the carrier $\mathrm{H}$ rotates, the angular velocity $\omega_{4}$ of the ring gear 4 is the same for both cases and is

$$
\omega_{B}=\omega_{4}=\frac{\omega_{A}}{i_{0}}=\frac{\omega_{A}}{\frac{z_{4}}{z_{1}}}=0.5 \omega_{A} .
$$




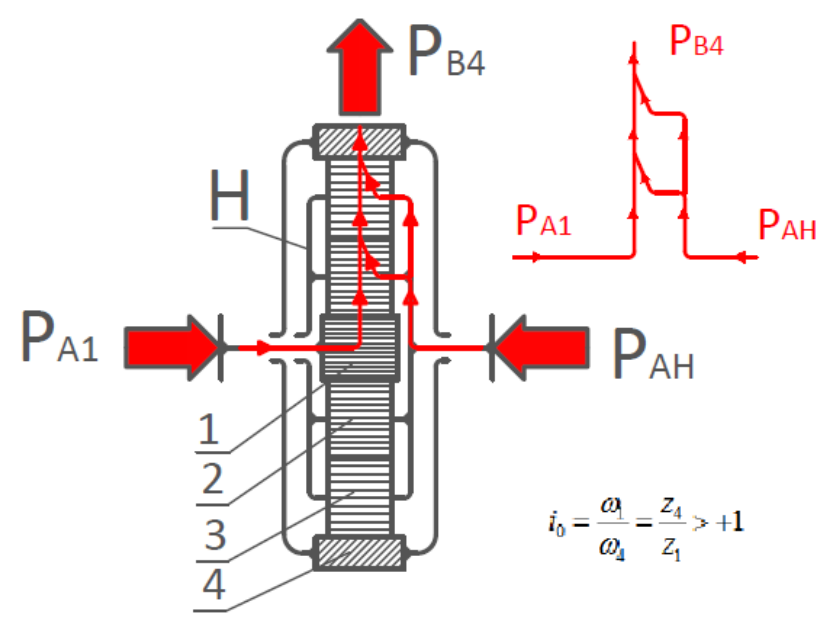

Fig. 6. $\overline{\mathrm{AAI}}-$ planetary gear train working as a differential.

It can be seen that the kinematics of this gear train at $\mathrm{i}_{0}=+2$ is the same as that of $\overline{\mathrm{AAA}}$ and $\overline{\mathrm{IAI}}-$ gear trains. Since at $i_{0}=+2$ the acting external torques of the sun gear 1 and of the carrier $\mathrm{H}$ are the same, i.e.

$$
T_{1}=T_{H}
$$

as well as the angular velocities of the two motors and of the elements driven by them - the sun gear 1 and the carrier H, i.e..

$$
\omega_{1}=\omega_{H}=\omega_{A} .
$$

then it follows that both motors have the same power

$$
P_{1}=P_{H}
$$

It is the same with $\overline{\mathrm{AAA}}$ and $\overline{\mathrm{IAI}}$ - gear trains, when $i_{0}=-1$, but not with $\overline{\mathrm{AI}}$ - gear train, where $i_{0} \neq-1$.

The considered $\overline{\mathrm{AAI}}$ - gear train has the advantage that axially it is very compact in comparison with the previous $\overline{\mathrm{AI}}$ - gear train. However, it has more gear wheels as compared to the $\overline{\mathrm{AI}}$ - gear train which means more complicated and more expensive production, as well as a problem with the internal load distribution [1].

\section{Compound planetary gear train}

Up to now the cases of simple, i.e. single-carrier planetary gear trains working as a differential were examined. As it can be seen on Fig. 7, the compound planetary gear trains, i.e. those with $\mathrm{F}=2$ degrees of freedom, can also work as a differential [4]. In the case shown the gear train is composed by the two of the most common type of planetary gear trains, namely, the $\overline{\mathrm{AI}}-$ gear train (Fig. 5).

Since the gear train is not simple, but a compound one, its analysis can be made in a different way, using the acing powers - the input power $P_{A}$ and the output power $P_{B}$.

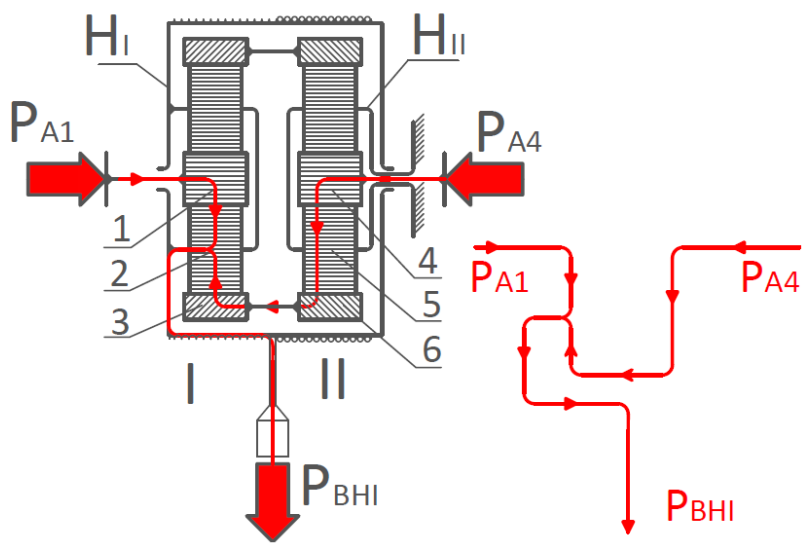

Fig. 7. Compound planetary gear train working as a differential.

For this purpose the following assumptions are made here:

- The two compound gear trains are kinematically the same, i.e. their basic speed ratios $i_{O I}$ and $i_{O I I}$ are the same

$$
i_{0 I}=-\frac{z_{3}}{z_{1}}=i_{0 I I}=-\frac{z_{6}}{z_{4}}=i_{0}=-3
$$

- The angular velocities of the two motors I and II, driving the two central gear wheels 1 and 4 are the same, and these are the input angular velocities

$$
\omega_{A I}=\omega_{A 1}=-\omega_{A I I}=\omega_{A 4}=\omega_{A}
$$

What is peculiar in this case is that the motors rotate in opposite directions.

It is also assumed that their torques acting on the two sun gear 1 and 4 are the same, and moreover, for convenience they are considered as equal to 1

$$
T_{A I}=T_{1}=T_{A I I}=T_{4}=T_{A}=+1 .
$$

The torques acting on the two carriers $\mathrm{H}_{\mathrm{I}}$ and $\mathrm{H}_{\mathrm{II}}$ are the same and with the accepted basic speed ratio of the two composing gear trains $i_{0}=-3$, these are

$$
T_{H I}=T_{H I I}=T_{B}=-4 \text {. }
$$

In addition, the output torque $T_{B}=T_{H}$, because only the carrier $\mathrm{H}_{\mathrm{I}}$ rotates, and the carrier $\mathrm{H}_{\mathrm{II}}$ is still.

When the gear train works with the two driving shafts, and the sun gears 1 and 4 are the input elements, and the carrier $\mathrm{H}_{\mathrm{I}}$ is the output element, then for the three acting on the gear train powers (input $P_{A I}$ and $P_{A I I}$ and output $P_{B}$ ) we can write:

$$
\begin{aligned}
\sum P_{i} & =P_{A I}+P_{A I I}+P_{B}= \\
& =T_{A I} \cdot \omega_{A I}+\left(-T_{A I I}\right)\left(-\omega_{A I I}\right)+T_{B} \cdot \omega_{B}= \\
& =2 T_{A} \cdot \omega_{A}+T_{B} \cdot \omega_{B}=0
\end{aligned}
$$

where for $\omega_{\mathrm{B}}$ it follows that 


$$
\omega_{B}=\frac{-2 T_{A} \cdot \omega_{A}}{T_{B}}=\frac{-2 \cdot 1 \cdot \omega_{A}}{-4}=+0.5 \omega_{A} .
$$

When only the left motor works which drives the sun gear 1 with still ring gear 3 , for $\omega_{B}$ directly follows that

$$
\omega_{B}=\frac{\omega_{A}}{1-i_{0}}=\frac{\omega_{A}}{1-(-3)}=\frac{\omega_{A}}{4}=+0.25 \omega_{A} .
$$

For the third case, when only the right motor rotates, which drives the sun gear 4 with still carrier $\mathrm{H}_{\mathrm{II}}$, from the summation of the powers it follows that

$$
\sum P_{i}=P_{A I I}+P_{B}=\left(-T_{A I I}\right)\left(-\omega_{A I I}\right)+T_{B} \cdot \omega_{B}=0
$$

and

$$
\omega_{B}=-\frac{T_{A} \cdot \omega_{A}}{T_{B}}=-\frac{1 \omega_{A}}{-4}=+0.25 \omega_{A}
$$

i.e. in this case the same output angular velocity $\omega_{B}$ is obtained.

If the two motors start rotating unidirectionally, then the output element the carrier $\mathrm{H}_{\mathrm{I}}$ remains still, i.e. $\omega_{H I}=0$ and no power is transmitted.

\section{Conclusion}

The paper considers the gear train types which are suitable for work as differentials. With a one-motor drive one differential works as a division gear train and with a two-motor drive - it works as a summation gear train. Both types of differentials are used in practice. They provide maximum security in particularly responsible drives. The knowledge of the wide capabilities of the planetary gear trains in general and in particular their work as differentials provides the constructor with expertise in drives better possibilities for successful technical solutions.

\section{References}

1. K. Arnaudov and D. Karaivanov. Planetary gear trains (CRC Press, Boca Raton, 2019)

2. K. Arnaudov, D. Karaivanov, and S. Troha. Fillet curve of the internal toothing - features, hazards, significance. Proceedings of the $5^{\text {th }}$ International Conference on Power Transmissions BAPT2016, Ohrid, Macedonia. 15-22 (2016)

3. K. Arnaudov, D. Karaivanov, A. Dobreva, and V. Ivanov. Involute internal meshing (TU-Sofia Press, Sofia, 2019)

4. J. Looman. Zahnradgetriebe - Grundlagen, Konstruktion, Anwendung in Fahrzeugen. 3. Auflage, Berlin: Springer-Verlag (1996) 